\title{
$B$ - $B O X$ genes: genome-wide identification, evolution and their contribution to pollen growth in pear (Pyrus bretschneideri Rehd.)
}

(1) CrossMark

\author{
Yunpeng Cao ${ }^{1}$, Yahui Han², Dandan Meng ${ }^{1}$, Dahui Li ${ }^{1}$, Chunyan Jiao ${ }^{1}$, Qing Jin ${ }^{1}$, Yi Lin $^{1}$ and Yongping Cai ${ }^{* *}$
}

\begin{abstract}
Background: The B-BOX (BBX) proteins have important functions in regulating plant growth and development. In plants, the $B B X$ gene family has been identified in several plants, such as rice, Arabidopsis and tomato. However, there still lack a genome-wide survey of $B B X$ genes in pear.

Results: In the present study, a total of 25 BBX genes were identified in pear (Pyrus bretschneideri Rehd.). Subsequently, phylogenetic relationship, gene structure, gene duplication, transcriptome data and qRT-PCR were conducted on these $B B X$ gene members. The transcript analysis revealed that twelve PbBBX genes (48\%) were specifically expressed in pear pollen tubes. Furthermore, qRT-PCR analysis indicated that both PbBBX4 and PbBBX13 have potential role in pear fruit development, while PbBBX5 should be involved in the senescence of pear pollen tube.

Conclusions: This study provided a genome-wide survey of $B B X$ gene family in pear, and highlighted its roles in both pear fruits and pollen tubes. The results will be useful in improving our understanding of the complexity of BBX gene family and functional characteristics of its members in future study.
\end{abstract}

Keywords: $B-B O X$, Systematic analysis, Pear, Relative gene expression

\section{Background}

Zinc-finger protein is one of the important transcription factors, which play important roles in plant growth, development and response to environmental changes. The zinc-finger protein whose three-dimensional is stabilized by binding zinc ions [1], could interact with DNA, RNA, and proteins involved in plant cell life activities [2]. $B$ $B O X$ gene family belongs to the zinc-finger protein family. In addition to the conserved B-BOX domain, some of the B-BOX members contain other family-specific domains, such as CCT (CONSTANS, CO-like and TOC1) domain. The $B-B O X$ gene family could be divided into five subfamilies according to the number of B-BOX domains or the CCT domain they contained [2]. After the first identification of the B-BOX member from Xenopus laevis [3], its ortholog (CONSTANS: $\mathrm{CO}$ ) in plant was cloned from Arabidoosis thaliana, with function in the photoperiod regulation of plant flowering time [4]. Subsequent

\footnotetext{
* Correspondence: swkx12@ahau.edu.cn

'School of Life Sciences, Anhui Agricultural University, Hefei 230036, China Full list of author information is available at the end of the article
}

researches further revealed that the B-BOX transcription factors in plants played very pivotal roles in mediating various life activities, such as seed germination [5], flowering [6], shade avoidance response [7], biological or abiotic stress response [8] and plant hormone signal transduction [9]. Recent studies have shown that as a negative regulator in brassinosteroid signaling pathways, BBX20 (AtBZS1) from $A$. thaliana could attach to $\mathrm{E} 2$ by recognition of COP1 (Constitutively Photomorphogenic 1), a key factor in light signal transduction, then for degradation by $26 \mathrm{~S}$ proteasomes [9]. Gangappa et al. (2013) stated that $A t B B X 25$ is involved in the negative regulation of plant photo-morphogenesis by forming a dimer with HY5 (Protein long hypocotyl 5) and suppressing its function [10]. Studies in apples found that MdBBX22 (MdCOL11) involved in the UV-B-induced synthesis of the anthocyanin synthesis [11]. Based on these results, it was implied that $B-B O X$ gene members might be associated with COP1-HY5-mediated optical signal transduction pathway, and further involved in plant light morphogenesis, regulating flowering, secondary metabolite synthesis and regulation of a variety of life activities. 
It has been proved that some transcription factor family should play an important role in the development of pear fruits, such as MYB and heat shock factor gene family [12, 13]. Two MYB family members, PbMYB25 and PbMYB52 were found to be the candidate genes involved in the regulation of lignin synthesis within pear fruits [12]. Although much knowledge on the function of $B-B O X$ gene family, such as responses to biotic and abiotic stresses and involvement in light signal transduction pathway, has been advanced [14-16], there is less research on their roles in pear pollen and fruit development. The completed pear genome sequencing [17] provided useful information for comprehensive analysis of the pear $B B X$ gene family. In this study, 25 nonredundant members were identified in the pear $B B X$ family. Subsequently, the detailed phylogenetic and expression pattern analyses of these $B B X$ genes were carried out. The present results will be useful for further functional characterization of $B B X$ genes in pear.

\section{Results}

Identification of $B B X$ genes in pear

To obtain BBX proteins in pear genome, the published Arabidopsis BBX proteins were employed as a query to search against the local pear genome database by using DNAtools software. After removing the redundant and repeated sequences, a total of 25 putative BBX protein sequences were confirmed in pear. For the sake of consistency, these $B B X$ genes were sequentially named after $P b B B X 1$ to $P b B B X 25$. The detailed information on the gene identifier, chromosome location, protein structure and the characteristics of the corresponding PbBBX proteins were listed in Table 1. The length of the amino acid sequence sequences ranged from 142 (PbBBX23) to 859 (PbBBX7). The pear $B B X$ genes encode proteins with predicted theoretical isoelectric points of 4.48-9.02 and molecular weights from 15.63900 (PbBBX23) to 93.60447 (PbBBX7) kDa (Table 1).

Table 1 The detailed information of PbBBX members

\begin{tabular}{|c|c|c|c|c|c|c|c|c|c|}
\hline Gene & Gene identifier & 5' End & 3' End & Chr & $\mathrm{AA}$ & pls & MW & Domains & Structure \\
\hline$P b B B \times 1$ & Pbr016562.1 & $17,929,143$ & $17,931,284$ & 17 & 397 & 5.41 & 43.92956 & $2 \mathrm{BBX}+\mathrm{CCT}$ & 1 \\
\hline PbBBX2 & Pbr023570.1 & $13,087,654$ & $13,089,051$ & 16 & 424 & 7.87 & 45.89379 & $2 \mathrm{BBX}+\mathrm{CCT}$ & I \\
\hline PbBBX3 & Pbr019957.1 & $5,731,876$ & $5,732,987$ & 15 & 340 & 5.84 & 37.97752 & $2 \mathrm{BBX}+\mathrm{CCT}$ & । \\
\hline PbBBX4 & Pbr036464.1 & $16,593,377$ & $16,594,915$ & 8 & 340 & 5.95 & 37.81046 & $2 \mathrm{BBX}+\mathrm{CCT}$ & । \\
\hline PbBBX5 & Pbr040252.1 & $20,855,304$ & $20,857,692$ & 17 & 490 & 6.54 & 53.16922 & $2 \mathrm{BBX}+\mathrm{CCT}$ & $\|$ \\
\hline PbBBX6 & Pbr022786.1 & $1,461,782$ & $1,463,416$ & 3 & 379 & 5.49 & 41.19483 & $2 \mathrm{BBX}+\mathrm{CCT}$ & $\|$ \\
\hline PbBBX7 & Pbr026954.1 & $6,184,619$ & $6,192,599$ & 13 & 859 & 5.2 & 93.60447 & $2 \mathrm{BBX}+\mathrm{CCT}$ & $\|$ \\
\hline PbBBX8 & Pbr038936.1 & $15,996,677$ & $15,998,414$ & 14 & 447 & 5.06 & 50.13728 & $1 \mathrm{BBX}+\mathrm{CCT}$ & III \\
\hline PbBBX9 & Pbr020281.1 & $3,789,123$ & $3,790,874$ & 6 & 459 & 5.26 & 51.07542 & $1 \mathrm{BBX}+\mathrm{CCT}$ & III \\
\hline PbBBX10 & Pbr013295.1 & $21,079,074$ & $21,081,045$ & 3 & 453 & 5.23 & 50.07548 & $1 \mathrm{BBX}+\mathrm{CCT}$ & III \\
\hline PbBBX11 & Pbr028831.1 & $2,106,467$ & $2,108,184$ & 13 & 454 & 5.45 & 50.59912 & $1 \mathrm{BBX}+\mathrm{CCT}$ & III \\
\hline$P b B B \times 12$ & Pbr022361.1 & $24,883,049$ & $24,886,946$ & 10 & 208 & 5.88 & 22.95886 & $2 \mathrm{BBX}$ & IV \\
\hline PbBBX13 & Pbr038976.1 & 52,556 & 56,089 & 5 & 199 & 5.68 & 22.02697 & $2 \mathrm{BBX}$ & IV \\
\hline PbBBX14 & Pbr042773.1 & $19,278,411$ & $19,280,758$ & 15 & 185 & 7.05 & 20.53247 & $2 \mathrm{BBX}$ & IV \\
\hline PbBBX15 & Pbr019591.1 & 648,141 & 649,024 & 5 & 222 & 6.34 & 24.86486 & $2 \mathrm{BBX}$ & IV \\
\hline$P b B B \times 16$ & Pbr015820.1 & $26,044,737$ & $26,045,718$ & 10 & 224 & 5.92 & 25.04003 & $2 B B X$ & IV \\
\hline PbBBX17 & Pbr005884.1 & $2,824,859$ & $2,826,234$ & 15 & 302 & 6.82 & 33.21935 & $2 \mathrm{BBX}$ & IV \\
\hline$P b B B \times 18$ & Pbr020473.1 & $1,098,702$ & $1,100,900$ & 11 & 288 & 5.47 & 30.95676 & $2 \mathrm{BBX}$ & IV \\
\hline PbBBX19 & Pbr032616.1 & $3,755,943$ & $3,757,523$ & 9 & 243 & 5.29 & 26.51099 & $2 \mathrm{BBX}$ & IV \\
\hline PbBBX20 & Pbr034751.1 & $1,633,105$ & $1,635,052$ & 17 & 242 & 5.09 & 26.64001 & $2 \mathrm{BBX}$ & IV \\
\hline$P b B B \times 21$ & Pbr033352.1 & $41,552,552$ & $41,553,827$ & 15 & 246 & 4.55 & 26.4555 & $1 \mathrm{BBX}$ & V \\
\hline$P b B B \times 22$ & Pbr011255.1 & $22,264,853$ & $22,266,136$ & 17 & 249 & 4.48 & 26.96689 & $1 \mathrm{BBX}$ & V \\
\hline PbBBX23 & Pbr000255.1 & $26,681,876$ & $26,682,798$ & 5 & 142 & 4.51 & 15.639 & $1 \mathrm{BBX}$ & V \\
\hline$P b B B \times 24$ & Pbr022252.1 & $14,907,160$ & $14,908,704$ & 17 & 270 & 8.93 & 29.41226 & $1 \mathrm{BBX}$ & V \\
\hline$P b B B \times 25$ & Pbr031832.1 & $19,005,388$ & $19,007,067$ & 15 & 271 & 9.02 & 29.72965 & $1 \mathrm{BBX}$ & V \\
\hline
\end{tabular}


Protein sequence and phylogenetic analysis of the pear $B B X$ gene family

The identified $\mathrm{PbBBX}$ proteins showed a wide variation of molecular length ranged from 142 to 859 amino acids. Out of 25 PbBBXs, seven were found to contain a conserved CCT domain and two B-BOX domains. Four and five members contained one B-BOX plus a CCT domain, or only one $\mathrm{B}-\mathrm{BOX}$ domain, respectively, with the remaining nine members containing two B-BOX domains (Table 1). The conserved structures of PbBBX members, were found with B-Box 1 sequence (CDXCXXXXAXVYC XADEAALCXXCDXXVHXANKLAXRHXH, X represents any amino acid) and B-Box 2 (CDICXXXXAXXXCXXD XAXLCXXCDXXVHXXXXXXHXRXXL) (Fig. 1). Additionally, the CCT domain was highly conserved among the PbBBXs (Fig. 1). The logos of these domains, including B-BOX1, B-BOX2 and CCT domain, were illustrated in Fig. 1, as well as the correspondence positions shown in Fig. 2.

To gain further insights into the phylogenetic relationship and divergence of the $B B X$ family, the phylogenic tree, including BBXs from Brachypodium distachyon, Oryza sativa, A. thaliana, Populus trichocarpa and pear, was constructed. Based on the phylogenetic analysis, this tree could be divided into five clades, and consistent with the previous studies [2, 18]. As shown in Fig. 3, most BBX members from poplar, Arabidopsis and pear were more closely than pear and Oryza sativa, Brachypodium distachyon. Among them, the members from clades I, II, III, contained two B-BOX domains plus a CCT domain, implying that these genes contained
CCT-domain might play a crucial role in the control of flowering $[4,19]$. On the contrary, the members from clade VI and clade V lacked CCT-domain and only contained one or two B-BOX domain. Previous studies have shown that the B-BOX domains $\left(\mathrm{CX}_{2} \mathrm{CX}_{8} \mathrm{CX}_{7} \mathrm{CX}_{2} \mathrm{CX}_{4} \mathrm{HX}_{8} \mathrm{H}\right)$ in the $\mathrm{N}$-terminal region, and the conserved Cysteine $(\mathrm{C})$ and Histidine $(\mathrm{H})$ residues in $\mathrm{B}-\mathrm{BOX}$ domain are predicted to be crucial for $\mathrm{BBX}$ protein-protein [2]. Interestingly, the Cysteine $(\mathrm{C})$ and Histidine $(\mathrm{H})$ residues was also found to be conserved at $\mathrm{B}-\mathrm{BOX}$ domain in the $\mathrm{C}$-terminal region of the BBX members from clades I, II and IV, respectively (Fig. 1). In summary, the structure analyses of theses BBOX proteins were basically consistent with the phylogenetic relationship.

\section{Gene structure and gene duplication}

The previous studies implied that gene structural diversity can lead to the evolution of multi-gene families. To better characterize and understand the structural diversity of the $P b B B X$ genes, gene exon-intron analysis was carried out (Fig. 4). As shown in Fig. 4, the number of exons was ranged from 1 to 17 , with $P b B B X 7$ containing the highest amounts of exons (17) among the $P b B B X s$, 12 of $\mathrm{PbBBXs}$ containing two exons, and 3 only one exon, respectively. Additionally, pear $B B X$ genes were clustered in the same clade with the highly similar exonintron structure, for example, eight $\mathrm{PbBBX}$ s within the clades I and III (containing two exons), and most members belonging to clade IV (having three exons). Likewise, three genes in clade $\mathrm{V}$ only contained one exon, except for $P b B B X 24$ and $P b B B X 25$. These results

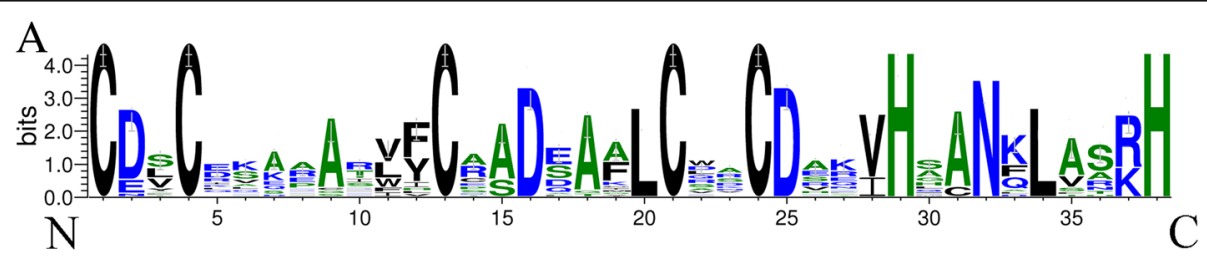

$\mathrm{B}$

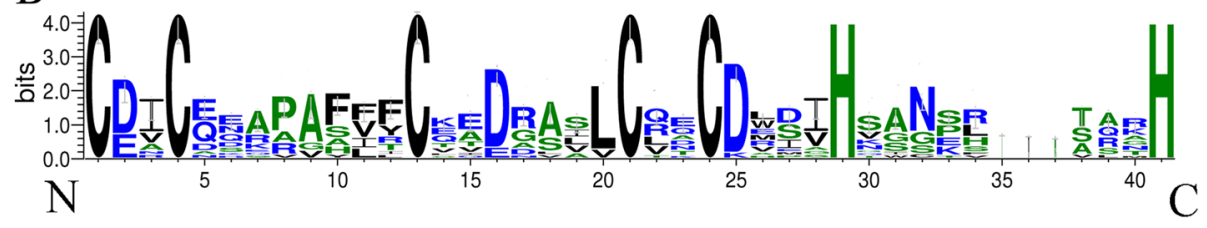

$\mathrm{C}$

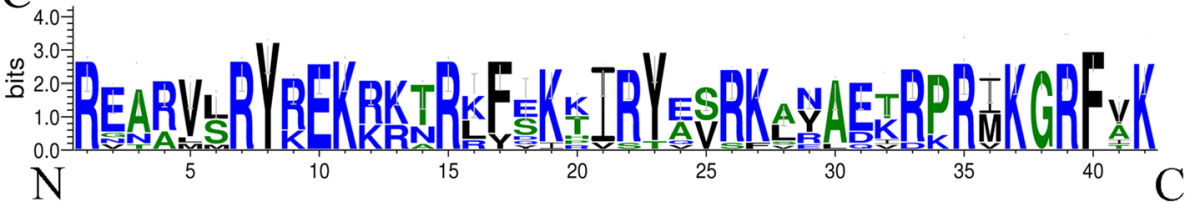

Fig. 1 Domain composition of PbBBX proteins. $\mathbf{a}, \mathbf{b}$ and $\mathbf{c}$ represent the protein alignment of the B-BOX 1, B-BOX 2 and CCT domain, respectively. The $x$-axis indicates the conserved sequences of the domain. The height of each letter indicates the conservation of each residue across all proteins. The $y$-axis is a scale of the relative entropy, which reflects the conservation rate of each amino acid 


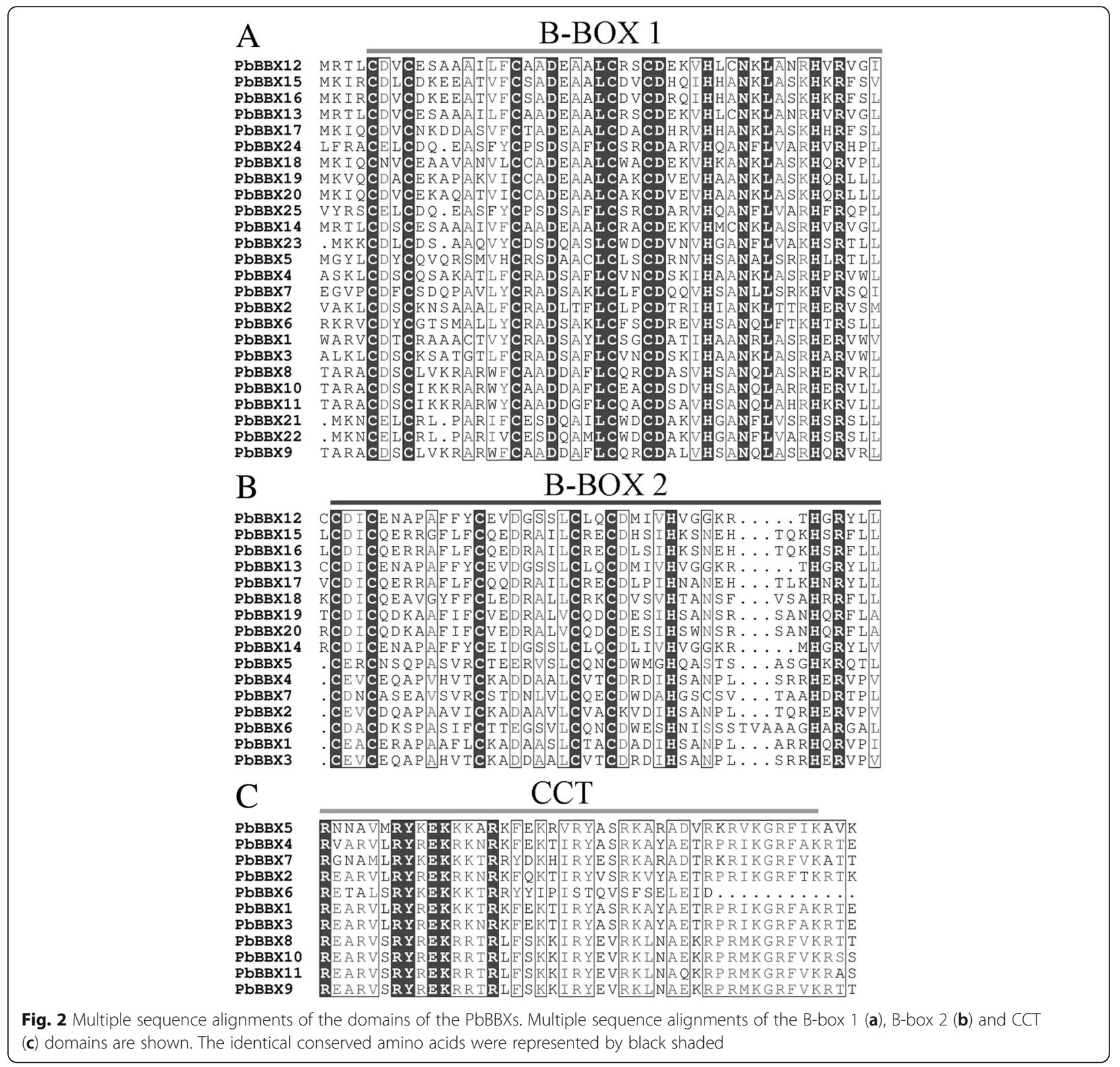

deduced that exon-loss or -gain had occurred during the evolution of the $P b B B X$ gene family and resulted in the functional divergence among the closely related PbBBXs (Fig. 4).

Up to data, the information about the expansion events of the $B B X$ gene family in pear was still unclear. To further reveal how $P b B B X$ genes were evolved, the chromosomal location and gene duplication events of $P b B B X$ genes were investigated. The chromosome locations and distributions of $25 \mathrm{PbBBX}$ genes were found among the 12 pear chromosomes (total of 17 chromosomes) (Additional file 1: Figure S1). Among them, the chromosomes 15 and 17 both contained highest number of $P b B B X$ genes (5); followed by chromosome 5 contained three genes; chromosomes 3 and 10 both had two genes; while the chromosomes $6,8,9,11,13,14$ and 16 only had one genes. Gene family expansion was usually achieved by tandem duplication and segmental duplication. In present study, we did not identify any of tandem duplication pairs. However, 13 segmental duplication gene pairs were found in pear genome by using MCScanX software (Fig. 5). Subsequently, the divergence time between these gene pairs was calculated with the period varied from 6.15 to 253.08 million years (Mya) (Additional file 2: Table S1).

To determine the selection pressure in duplication of $P b B B X$ genes, the non-synonymous (Ka)/synonymous (Ks) values were calculated for the 13 gene pairs. The 


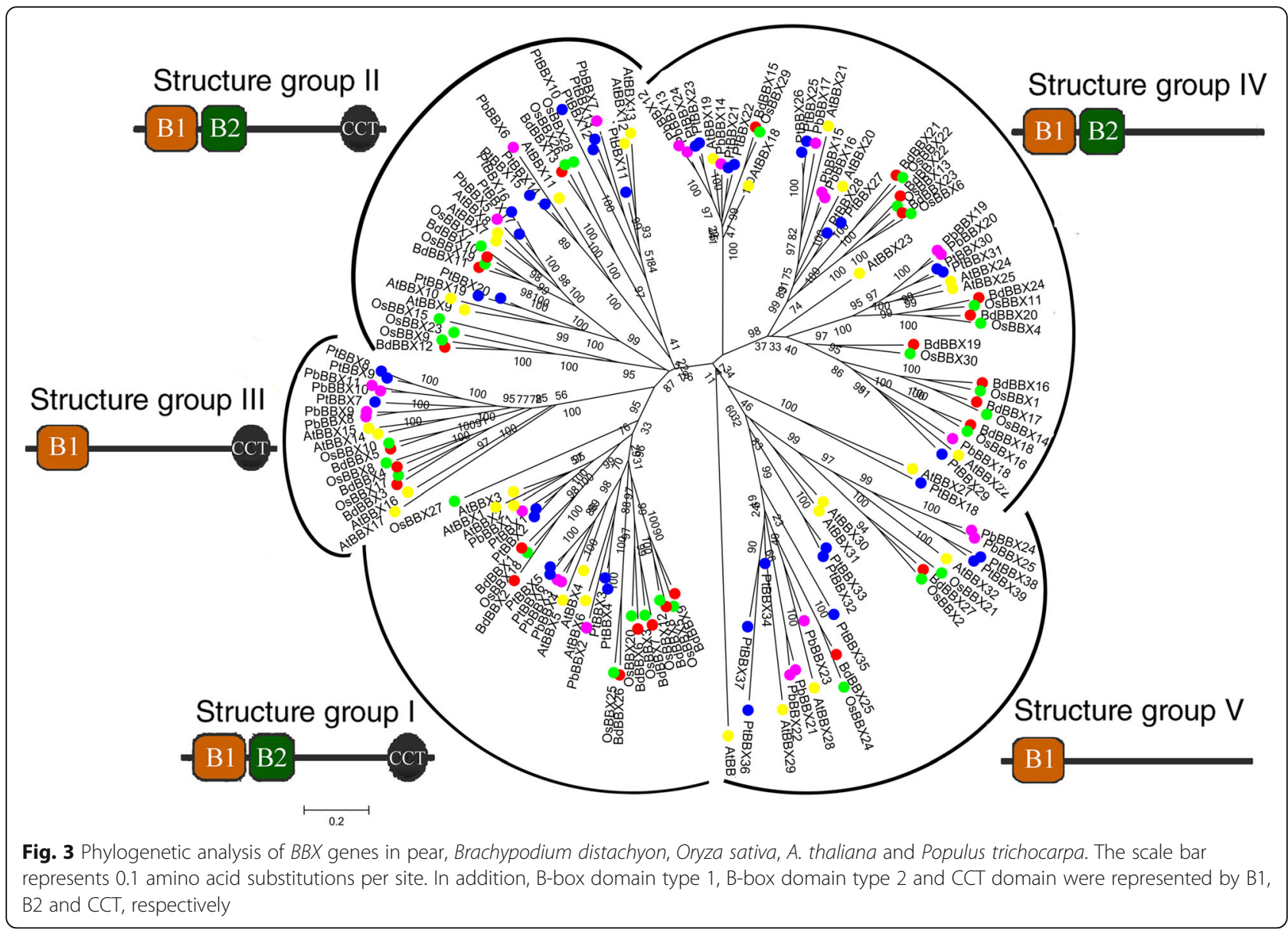

$\mathrm{Ka} / \mathrm{Ks}$ values of all $\mathrm{PbBBX}$ gene pairs were less than 1.0 (Additional file 2: Table S1), indicating that they were under strong purifying selective during their evolution and a conserved evolutionary pattern was shared among $B B X$ genes.

\section{Expression patterns of pear $B B X$ genes}

The pollen germination and pollen tube growth in many higher plants have been known to play a crucial role in sexual reproduction. Previous studies suggested that pollen tube via tip-growth rapidly extended and then underwent senescence within $15 \mathrm{~h}$ (P4: stopped-growth pollen tubes) in vitro [20]. In our study, to further understand the roles of $B B X$ family genes in pear pollen growth, expression patterns of $P b B B X$ genes were analyzed by transcriptome sequencing data. As shown in Figure (Additional file 3: Figure S2), 13 of $P b B B X$ genes (52\%) were not found to be expressed during the different developmental stages of pear pollen, implying that these genes might express in root, stem, leaf, or/and under special conditions. On the contrary, $12 \mathrm{PbBBX}$ genes (48\%) were detected to be expressed in a development-dependent pattern in pear pollen. For example, $5 \mathrm{PbBBX \textrm {s }}$ (PbBBX6, 7, 9, 11,12 ) were specifically expressed at P1 stage (mature pollen grains), while 2 ( $P b B B X 8$ and $P b B B X 10)$ at $\mathrm{P} 2$ stage (hydrated pollen grains) (Additional file 3: Figure S2). The high number of $P b B B X$ genes differentially expressed during pollen growth suggests that they are important proteins for signaling in this process. In addition, the expression patterns of $P b B B X$ genes were validated by using qRT-PCR during pear pollen tube growth (Additional file 4: Figure S3). We found that qRT-PCR results were almost consistent with that of transcriptome sequencing data, except $P b B B X 6$ and $P b B B X 7$. The reason for this divergence may be the lowest expression levels during pollen tube growth. Remarkably, compared with other periods, the expression levels of PbBBX5 gene reached its peak at P4 (stopped-growth pollen tubes), implying that this gene might play potential role in plant reproductive development, such as the senescence of pollen tubes.

Subsequently, the expression profiles of these $P b B B X$ genes in different tissues or organs were also surveyed by using qRT-PCR (Fig. 6). Results showed that except for $P b B B X s$ 6, 8, 9, 11, and 19 which showing no expression in the tested tissues or organs, PbBBXs 1, 2, 3, 4, 7, $10,14,16,18,20,21,22,23,24$ and 25 were predominantly expressed in leaves, while $P b B B X s 13$ and 17 were 


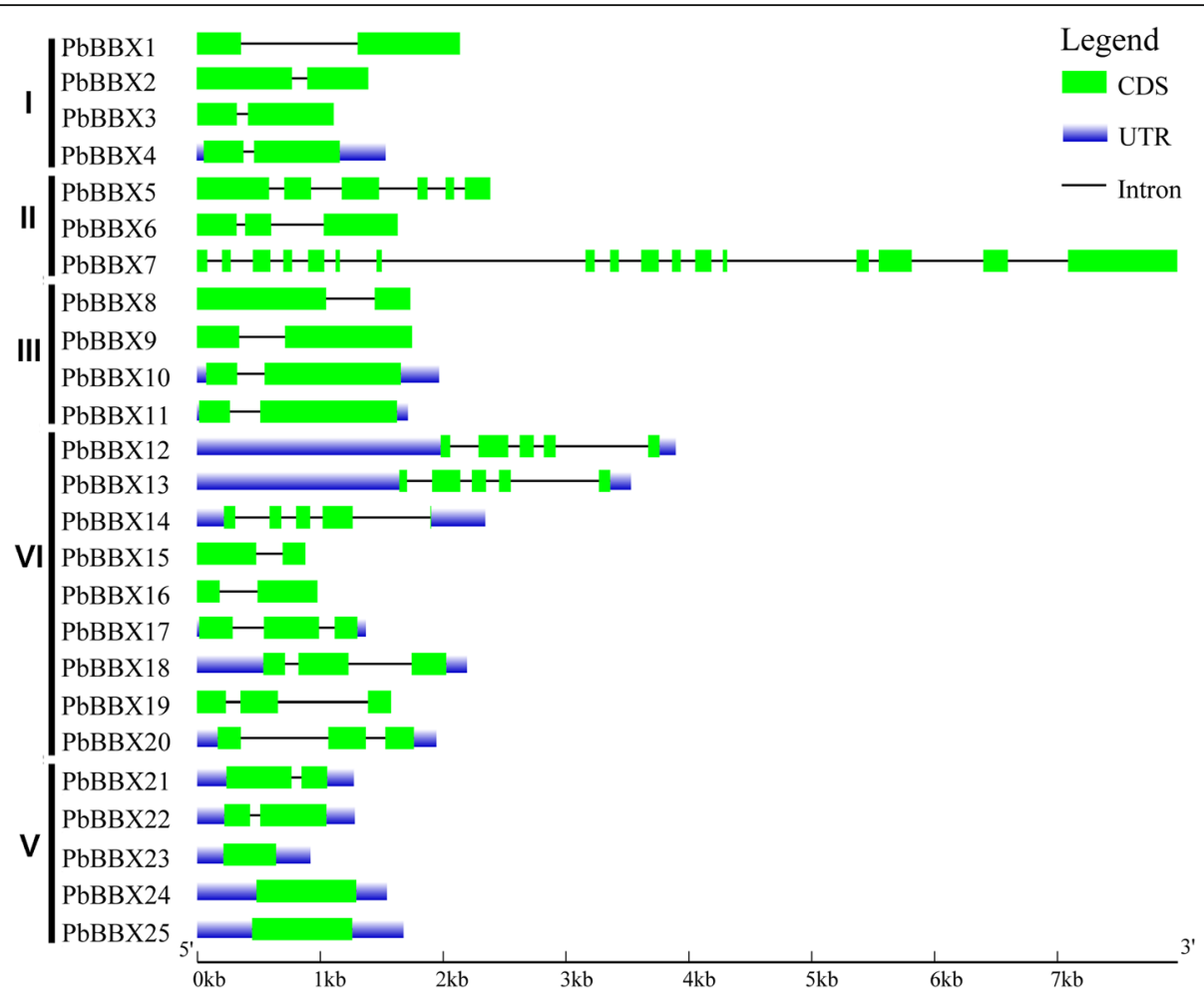

Fig. 4 Exon-intron structure of the PbBBX family generated from GSDS online website. Legend is at the top right of the Figure. The scale represents the length of the DNA sequence

highly expressed in roots. Furthermore, the expression levels of $P b B B X s$ 12, 17 and 25 was higher in root, stem or leaf than that those in different developmental stages of pear fruit. Interestingly, $P b B B X s 6,8,9$, and 11 were tissue-specifically expressed during different developmental stages of pear pollen. Additionally, we found $P b B B X 19$ was not expressed in root, stem, leaf and fruits, these results recommended that the functions need to be further studied at other tissues or special conditions (Fig. 6).

\section{Analysis of subcellular localization of $P b B B X 4, P b B B X 5$ and PbBBX13}

The nuclear localization of transcription factors is very important for its regulatory function. Previous studies reported that $\mathrm{BBX}$ proteins were predominantly located on the nucleus, such as SlBBX5, SlBBX7 and SlBBX15 in tomato [21]. The expression levels of $P b B B X 4, P b B B X 5$ and $P b B B X 13$ observed that they play an important role in the development of pear pollen tubes or fruits. To further understand these three proteins characteristics, subcellular localization experiment was carried out. Subsequently, we introduced GFP control and the PbBBX4-GFP, PbBBX5GFP and PbBBX13-GFP fusion constructs (Fig. 7a) by CaMV $35 S$ promoter into $N$. tabacum epidermal cells. As indicated in Fig. 7b, green fluorescence signals from the expressed fusion PbBBX4-GFP, PbBBX5-GFP and
PbBBX13-GFP were specifically distributed within the nuclei as confirmed by DAPI (DNA dye 4, 6-diamidino-2phenylindole) staining. However, the control GFP protein was observed throughout the whole cell (Fig. 7b). These results suggested that PbBBX4, PbBBX5 and PbBBX13 were nuclear proteins, and consistent with the previous results [21].

\section{Discussion}

Although the $B B X$ gene family has been identified in several model plants, such as rice [22], and Arabidopsis [2], its function and evolution was still unclear in pear. In this study, a comprehensive analysis of pear $B B X$ gene family was performed, including analyses of phylogeny, chromosome localization, gene duplication, sequence feature, and expression pattern.

A total of $25 B B X$ genes were identified from pear genome. The number of $B B X$ genes in pear was fewer, compared to their orthologs in tomato (29) [21], Arabidopsis (32) [2], and rice (30) [22]. Noteworthy, the pear genome size $(512 \mathrm{Mb})$ [17] was larger than those of rice (403 Mb) [23] or Arabidopsis (125 Mb) [24], although smaller than the tomato genome size $(960 \mathrm{Mb})$ [25]. These results indicated that the $B B X$ gene family members may not be directly related to the genome sizes in different plants. Although the difference in number was not significant, however, the type of $B B X$ gene was 


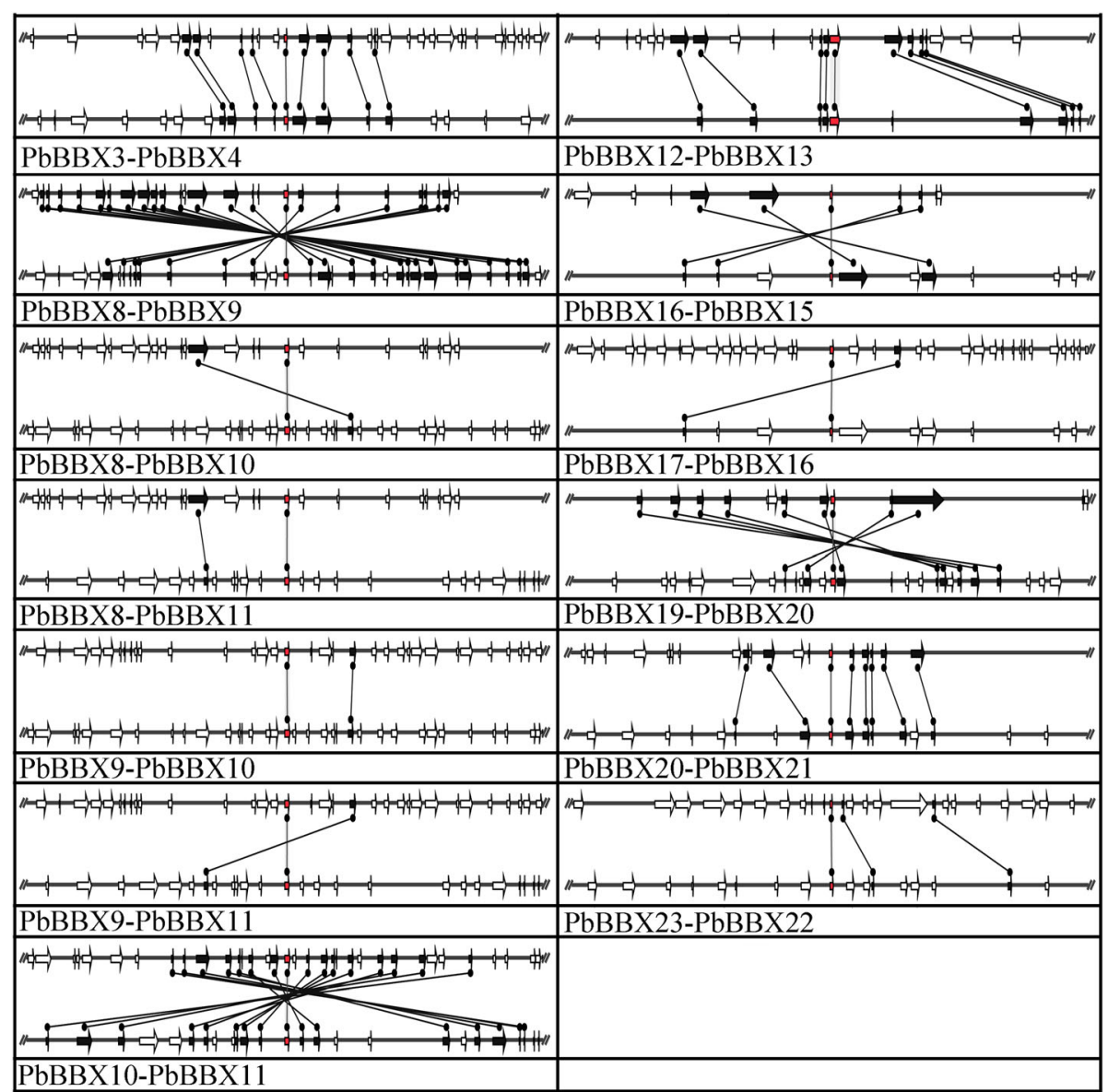

Fig. 5 Segmental duplication between members of the BBX gene family in pear. The map shows the $100 \mathrm{~kb}$ region on each side flanking of the BBX genes. The conserved gene pairs among the segments are connected with bands. Gene and its transcriptional orientation were represented by broad line with arrowhead

different among species. In tomato, the numbers of BBX members two tandem B-BOXes plus the CCT domain, BOX1 plus CCT, two tandem B-BOXes, and B-BOX1 only were 8, 5, 10 and 6, respectively [21]. In Arabidopsis, the corresponding numbers were $13,4,8$, and 7 [2]. And in pear were 7, 4, 9 and 6 . These results indicated that $B B X$ genes may have a common ancestor among different species, and were independently expanded after the divergence of the dicots and the monocots. In addition, to elucidate how the $B B X$ gene family evolved, a phylogenic tree of plant $B B X$ genes from monocots (rice and Brachypodium distachyum) and dicots (pear, poplar and Arabidopsis) was constructed. Within the phylogenic tree, $B B X$ s were divided into five clades: I, II, III, VI, and V. We found that most of the $B B X$ genes from the dicot were clustered together, implying that these genes might be orthologous genes as reported by previous studies $[2,18]$.

During the course of plant evolution, gene duplication plays an important role for generating novel genes. Gene duplication in plants has two main duplication patterns, including segmental duplication and tandem duplication [26], which had been demonstrated to play a key role in the expansion of gene family members in many species, such as the families WOX, MYB, PRX and $4 C L$ in pear [12, 27-29], the CHS in maize [30]. To further reveal the potential mechanism of evolution of the $B B X$ gene family, both the segmental and tandem duplication events were analyzed in pear. In present study, none of the $P b B B X$ genes were located in tandem. And 16 $P b B B X$ genes were identified to be arranged in segmental duplication regions of pear chromosomes. These results indicated that segment duplications were the main driver force for the expansion of pear $B B X$ gene family members. In addition, previous studies reported that tandem duplication often occurred in the large and rapidly evolving gene family, such as NBS-LRR gene family [31], whereas, segmental duplication usually occurred in the slowly evolving gene family, such as $M Y B$ gene family [31]. The present results indicated that pear $B B X$ gene family should be classified as a gene family with slow evolutionary characteristics. 


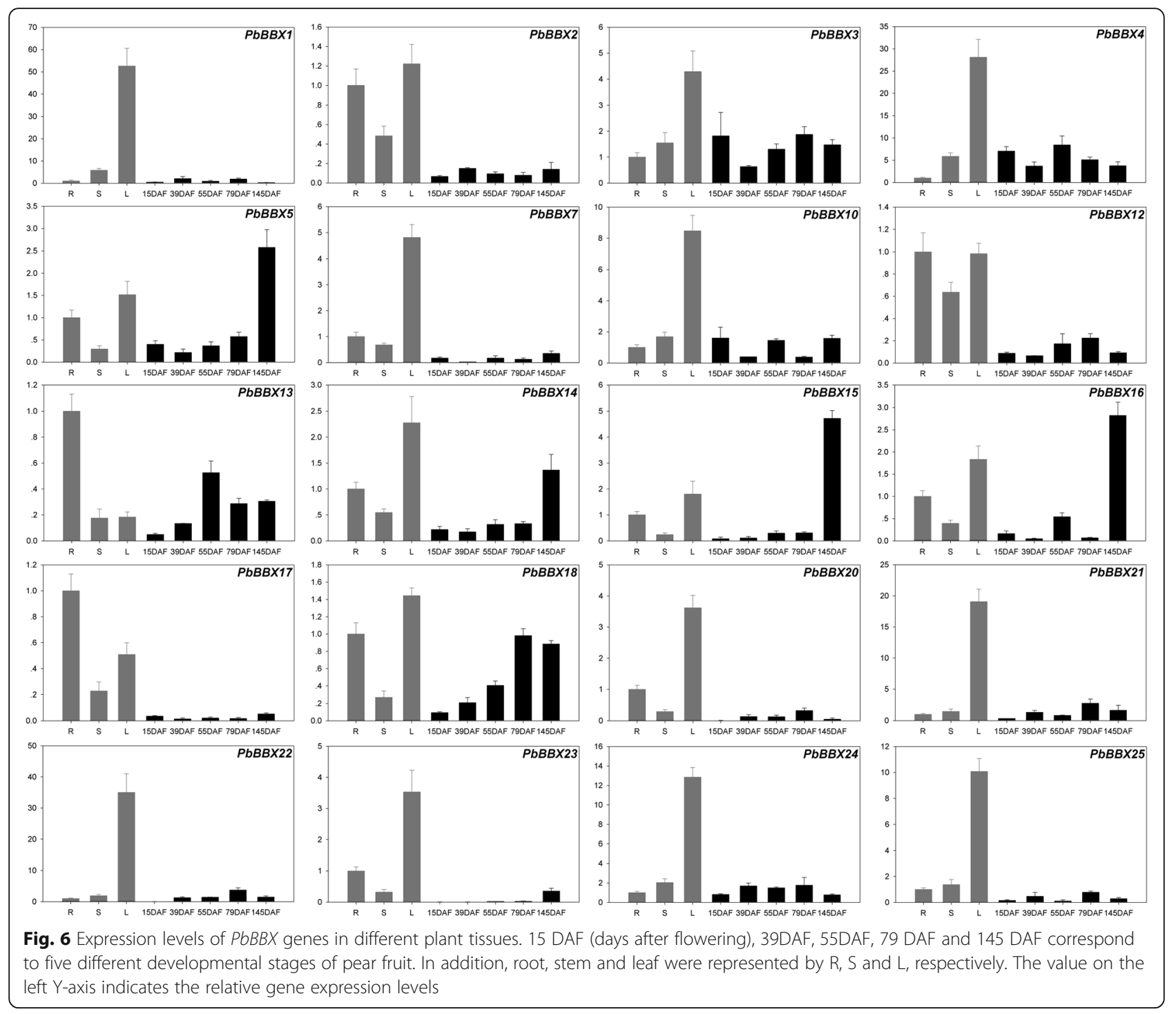

The collected transcriptome data showed that $B-B O X$ gene family was involved in the pollen tube growth. Twelve of the pear $B-B O X$ gene family members were found to be involved in the development of pear pollen tubes. The previous report by Gangappa et al. [32], has shown that during the photo-morphogenesis in Arabidopsis, some of BBX family members could competitively interact with protein and further regulate HY5 activity, leading to the fine regulation of the pollen tube development. Similarly, during the growth of pear pollen tube, the $B B X$ family members might regulate the development process. Altogether, our results suggested that this gene family was not only involved in the development of floral organs, but also in the development of pollen tubes. The latter might be fulfilled by several genes (PbBBX6,8,9, and 11) which were specifically expressed in pear pollen tubes. Previous studies by Gao et al. [20] suggested that as the extension of cultural duration to $15 \mathrm{~h}$ (P4: stopped-growth pollen tubes), pear pollen tubes growth became slow and exhibited some characteristics of senescence at $\mathrm{P} 4$ post-cultured in vitro, implying that the senescence of the pear pollen tubes might occur at the P4 period. In the present study, the expression level of $P b B B X 5$ in the pear pollen tubes was significantly increased, and its expression pattern was basically consistent with the previous report [20]. The increased expression level of $P b B B X 5$ suggested that it might play a role in the regulation of pollen tube senescence.

Additional, the expression patterns of both $P b B B X 4$ and $P b B B X 13$, were consistent with the content dynamics of fruit lignin: at the early to middle stages the concentration of these contents increased, while at the mature stage showed less concentration [27, 33, 34]. These results suggested that these two genes might regulate the lignin synthesis in pear fruits. As reported 


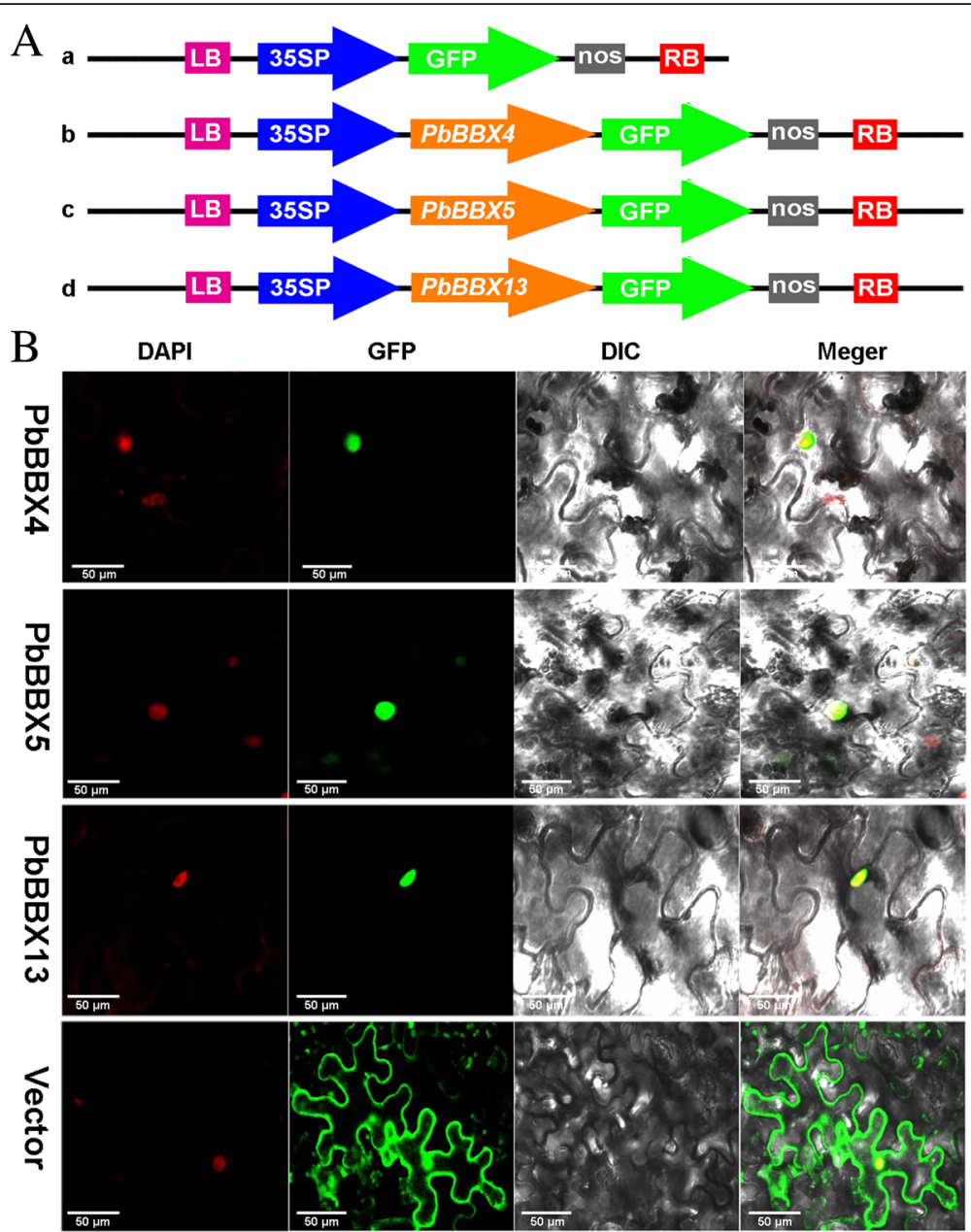

Fig. 7 Subcellular localization of PbBBX4-GFP, PbBBX5-GFP and PbBBX13-GFP fusion protein. a Schematic representation of the 35S: GFP, 35S: PbBBX4-GFP, 35S: PbBBX5-GFP and 35S: PbBBX13-GFP fusion constructs used for transient expression. $\mathbf{b}$ The three PbBBX-GFP fusion proteins (PbBBX4-GFP, PbBBX5-GFP, and PbBBX13-GFP) as well as GFP as the control, were transiently expressed in tobacco leaves and observed by fluorescence microscopy. Bars $=50 \mu \mathrm{m}$

by our previous studies, the content of stone cells was thought to be an important factor affecting the quality of pear fruit [33, 34]. Due to closely relationship between the development of stone cell and the biosynthesis of lignin $[33,34]$, it is possible that $P b B B X 4$ and $P b B B X 13$ could be applied for the improvement of pear fruits using genetic engineering.

\section{Conclusions}

In the present study, a systematic analysis of the $P b B B X$ gene family was carried out, including conserved domain, gene structure, phylogenetic relationship, chromosome location, gene duplication and expression pattern analysis. The $P b B B X$ genes were divided into five clades: I (4 genes), II (4 genes), III (3 genes), IV (9 genes), V (5 genes), which were supported by gene structural and conserved domain analysis. Gene duplication analysis suggested that the segmental duplications have driven expansion of the pear $B B X$ gene family. Transcriptome sequencing and qRT-PCR analysis revealed that the $P b B B X$ genes play an important role in different pollen tube and fruit developmental stages. Further analysis revealed that $\mathrm{PbBBX} 4$ and $P b B B X 13$ might regulate the synthesis of pear fruit lignin, and PbBBX5 might play a role in the senescence of pollen tubes.

\section{Methods}

\section{Sequence retrieval}

To identify and annotate $B B X$ genes in pear, the Arabidopsis BBX protein sequences [2] from the Arabidopsis Information Resource (TAIR) database (http://www.arabidopsis.org) were used as queries to search against pear genome database with BLASTP program (e-value $<1 \mathrm{e}-5)$. Subsequently, the putative $B B X$ genes in pear genome, 
were verified for the presence of the B-BOX domain by screening against the InterProScan (http://www.ebi.ac.uk/ Tools/pfa/iprscan/) [35], Pfam (http://pfam.sanger.ac.uk/) [36] and SMART (http://smart.embl-heidelberg.de/) [37] database.

\section{Phylogenetic analysis and sequence alignment}

Multiple sequence alignments of the 25 pear BBX proteins were generated using ClustalW version 1.83 with default settings, and the neighbor-joining $(\mathrm{NJ})$ tree was constructed by MEGA 5.2 with bootstrap analysis (1000 replicates) [38]. The pfam (http://pfam.xfam.org) [36], InterProscan (http://www.ebi.ac.uk/interpro/scan.html) [35], and SMART (http://smart.embl-heidelberg.de) [37] were used to identify domains. The sequence logos of conserved domains were generated using online WebLogo (http://weblogo.berkeley.edu/logo.cgi) [39].

\section{Gene structure, chromosomal location, and duplication analysis}

The exons and introns of the $B B X$ genes were identified according to the pear genome annotation file. And exonintron map was generated by using Gene Structure Display Server (http://gsds.cbi.pku.edu.cn/) [40]. The chromosome location image of the pear $B B X$ genes on chromosomes or scaffolds was drawn using the MapInspect software according to the physical positions on the pear chromosomes. The MCScanX software (http://chibba.pgml.uga.edu/ mcscan2/) [41] was used to identify the duplications of PbPRXs. The Calculator 2.0 software [42] was used to estimate the nonsynonymous (Ka) and synonymous (Ks) substitution rates of the different gene duplication pairs. The Ks values were used to estimate the approximate date of every duplicated event occurred in pear, seeing the formula: $\mathrm{T}=\mathrm{Ks} / 2 \lambda \times 10^{-6}$ Mya $\left(\lambda=6.5 \times 10^{-9}\right)[17,43]$.

\section{Plant material}

The samples were collected from ten of healthy, 40-yearold pear trees (Pyrus bretschneideri cv. Dangshan Su), which have been managed under the same irrigation and fertilization in the orchard at Dangshan County, Anhui province, China. These pear samples under the same developmental period were grown toward the middle southern direction and collected on early April, 2016. Roots, stems, and leaves were collected at the fifteen the day after flowering (DAF). 40 fruits with the uniform size were collected on 19th April (15 DAF), 14 May (39 DAF), 30 May (55 DAF), 22 June (79 DAF), and 29 August (145 DAF) in 2016, respectively. The methods for collection and drying, and in vitro culture of pear pollen grains, were based on the procedures by Zhou. et al. 2016 [44].

\section{RNA-seq expression analysis}

The raw RNA-seq reads from pear pollen were download from the NCBI database (PRJNA299117) [44]. The pear pollen samples were as follows: P1: mature pollen grains, P2: hydrated pollen grains, P3: growing pollen tubes, and P4: stopped-growth pollen tubes. The analysis of raw RNA-seq data was according to previous method [45], and the RPKM (Reads Per Kilobase per Million mapped reads) values were used to estimate the gene expression level. The heatmap of $P b B B X$ genes was exhibited using $\mathrm{R}$ software (http://www.bioconductor.org/).

\section{qRT-PCR analysis}

The TIANGEN RNAprep pure (Tiangen, Beijing, China) was used to extract the total RNA according to the manufacturer's instructions, followed by DNaseI (Tiangen, Beijing, China) digestion to eliminate any contaminating DNA. For qRT-PCR analysis, the first-strand cDNAs was synthesized from the $1 \mu \mathrm{g}$ RNA using the Reverse Transcriptase M-MLV System (Tiangen, Beijing, China) according to the manufacturer's instructions. The Beacon Designer 7 software was used to design and check the gene-specific primers (Additional file 5: Table S2). The pear tubulin gene (forward primer: 5' -AGAACAAGAAC TCGTCCTAC-3'; reverse peimer: 5'-GAACTGCTCGCT CACTCTCC-3') was used as reference gene [46]. The qRT-PCR was carried out using SYBR Premixm Ex Taq ${ }^{\text {ma }}$ (TaKaRa, Japan) with the CFX96 Touch $^{\text {Tw }}$ Real-Time PCR Detection System (Bio-Rad, USA). For each sample, we executed three biological replicates. The $2^{-\Delta \Delta C T}$ method was used to estimate the relative expression level [47].

\section{Subcellular localization analysis}

The expression vectors of 35S:PbBBX5-GFP, 35S: PbBBX4GFP, and PbBBX13-GFP were constructed by insertion of cDNA PbBBX5, PbBBX4 and PbBBX13, into pCAMBIA1304 vector, respectively. After electroporation of these construction into Agrobacterium tumefaciens EHA105, using pCAMBIA1304 vector as negative control [48], the transformed bacterial cells were infected into the leaf tissue of Nicotiana tabacum as the method described by Sparkes et al. (2006) [49]. The transient expression of PbBBX-GFP was observed using a laser confocal microscope (Zeiss LSM700, Germany), the DNA dye 4,6-diamidino-2-phenylindole (DAPI) was used to visualize the nucleus.

\section{Additional files}

Additional file 1: Figure S1. Chromosomal locations and duplication events of $B B X$ genes in the pear genome. The chromosome number is implied at the top of each chromosome. The segmental duplicated genes are connected by color lines and marked by corresponding color boxes. The scale on the left represents the megabases (Mb). (TIFF $3910 \mathrm{~kb}$ ) 
Additional file 2: Table S1. $\mathrm{Ka} / \mathrm{Ks}$ analysis and divergence time estimated for pear duplicated BBX paralogs. (XLSX $11 \mathrm{~kb}$ )

Additional file 3: Figure S2. Expression profiles of $P b B B X$ genes in pear pollen. P1, P2, P3, and P4 are represents mature dry pollen, hydrated pollen grains, pollen tubes, and stop growing pollen tubes, respectively. Color scale represents log2 transformed RPKM (Reads Per Kilobase per Million mapped reads) values. The gradually change of the color indicates different expression level of PbBBX genes, and the middle expression level was represented by white color. (TIFF $87 \mathrm{~kb}$ )

Additional file 4: Figure S3. The gene expression of 12 PbBBX genes during pollen tube growth by qRT-PCR. The value on the left Y-axis indicates the relative gene expression levels. P1 (mature pollen grains), P2 (hydrated pollen grains), P3 (growing pollen tubes), and P4 (stopped-growth pollen tubes) correspond to four different developmental stages of pollen and pollen tube. (TIFF 123 kb)

Additional file 5: Table S2. Primers in this study. (XLSX $11 \mathrm{~kb}$ )

\section{Abbreviations}

BBX: B-BOX; COP1: Constitutively Photomorphogenic 1; DAF: Days after flowering; DAPI: DNA dye 4,6-diamidino-2-phenylindole; HMM: Hidden Markov Model; HY5: Protein long hypocotyl 5; Ka: Nonsynonymous; Ks: Synonymous; MEME: Multiple Em for Motif Elicitation; Mya: Million years; NJ: Neighbor joining; qRT-PCR: Real-Time PCR; RPKM: Reads Per Kilobase per Million mapped reads

\section{Acknowledgments}

We would like to thank Muhammad Abdullah for his careful reading and helpful comments on this manuscript. We extend our thanks to the reviewers and editors for their careful reading and helpful comments on this manuscript.

\section{Funding}

This study was supported by The National Natural Science Foundation of China (grant 31,640,068) and 2017 Graduate innovation fund of Anhui Agriculture University (2017yjs-31). The Funding bodies were not involved in the design of the study and collection, analysis, and interpretation of data and in writing the manuscript.

\section{Availability of data and materials}

RNA-seq data for expression profiles from this paper were downloaded from NCBI database (accession numbers: PRJNA299117) (https://

www.ncbi.nlm.nih.gov//bioproject/PRJNA299117). The genome sequence of pear was obtained from GigaDB database (http://gigadb.org/site/index). Pear $B B X$ gene IDs were listed in Table 1.

\section{Authors' contributions}

YCao and YCai designed and performed the experiments; YCao, DM, CJ and $\mathrm{YH}$ analyzed the data; YH, DM, DL, YL, QJ, YCao and YCai contributed reagents/materials/analysis tools; YCao wrote the paper. All authors reviewed and approved the final submission.

\section{Ethics approval and consent to participate}

The experiments did not involve endangered or protected species. No specific permits were required for these locations/activities because the pears used in this study were obtained from a horticultural field in Dangshan, which were demonstration orchards at Auhui Agricultural University.

\section{Consent for publication}

Not applicable.

\section{Competing interests}

The authors declare that they have no competing interests

\section{Publisher's Note}

Springer Nature remains neutral with regard to jurisdictional claims in published maps and institutional affiliations.

\section{Author details}

${ }^{1}$ School of Life Sciences, Anhui Agricultural University, Hefei 230036, China.

${ }^{2}$ State Key Laboratory of Tea Plant Biology and Utilization, Anhui Agricultural University, Hefei 230036, China.

Received: 31 March 2017 Accepted: 8 September 2017

Published online: 19 September 2017

\section{References}

1. Klug A, Schwabe JW. Protein motifs 5. Zinc fingers. Faseb J. 1995;9(8): 597-604.

2. Khanna R, Wu SH. The Arabidopsis B-Box zinc finger family. Plant Cell. 2009; 21(11):3416.

3. Torok $M$, Etkin LD. Two B or not two B? Overview of the rapidly expanding B-box family of proteins. Differentiation. 2001;67(3):63-71.

4. Putterill J, Robson F, Lee K, Simon R, Coupland G. The CONSTANS gene of Arabidopsis promotes flowering and encodes a protein showing similarities to zinc finger transcription factors. Cell. 1995;80(6):847.

5. Chang CS, Maloof JN, Wu SH. COP1-mediated degradation of BBX22/LZF1 optimizes seedling development in Arabidopsis. Plant Physiol. 2011;156(1):228-39.

6. Gonzálezschain ND, Díazmendoza M, Zurczak M, Suárezlópez P. Potato CONSTANS is involved in photoperiodic tuberization in a graft-transmissible manner. Plant J. 2012;70(4):678-90.

7. Crocco CD, Holm M, Yanovsky MJ, Botto JF. Function of B-BOX under shade. Plant Signal Behav. 2011;6(1):101-4.

8. Wang Q, Tu X, Zhang J, Chen X, Rao L. Heat stress-induced BBX18 negatively regulates the thermotolerance in Arabidopsis. Mol Biol Rep. 2013; 40(3):2679-88

9. Fan X-Y, Sun Y, Cao D-M, Bai M-Y, Luo X-M, Yang H-J, Wei C-Q, Zhu S-W, Sun $Y$, Chong K. BZS1, a B-box protein, promotes photomorphogenesis downstream of both brassinosteroid and light signaling pathways. Mol Plant. 2012:5(3):591-600.

10. Gangappa SN, Crocco CD, Johansson H, Datta S, Hettiarachchi C, Holm M, Botto JF. The Arabidopsis B-BOX protein BBX25 interacts with HY5, negatively regulating BBX22 expression to suppress seedling photomorphogenesis. Plant Cell. 2013;25(4):1243-57.

11. Bai S, Saito T, Honda C, Hatsuyama Y, Ito A, Moriquchi T. An apple B-box protein, MdCOL11, is involved in UV-B-and temperature-induced anthocyanin biosynthesis. Planta. 2014;240(5):1051-62.

12. Cao Y, Han Y, Li D, Lin Y, Cai Y. MYB transcription factors in Chinese Pear (Pyrus bretschneideri Rehd.): genome-wide identification, classification, and expression profiling during fruit development. Front Plant Sci. 2016;7(10):577.

13. Qiao X, Li M, Li L, Yin H, Wu J, Zhang S. Genome-wide identification and comparative analysis of the heat shock transcription factor family in Chinese white pear (Pyrus bretschneideri) and five other Rosaceae species. BMC Plant Biol. 2015:15(1):12.

14. Datta S, Hettiarachchi GH, Deng XW, Holm M. Arabidopsis CONSTANS-LIKE3 is a positive regulator of red light signaling and root growth. Plant Cell. 2006;18(1):70-84.

15. Sánchez JP, Duque P, Chua NH. ABA activates ADPR cyclase and CADPR induces a subset of ABA-responsive genes in Arabidopsis. Plant J. 2004; 38(3):381-95.

16. Hannah MA, Heyer AG, Hincha DK. A global survey of gene regulation during cold acclimation in Arabidopsis thaliana. PLoS Genet. 2005;1(2):e26.

17. Wu J, Wang Z, Shi Z, Zhang S, Ming R, Zhu S, Khan MA, Tao S, Korban SS, Wang $H$. The genome of the pear (Pyrus bretschneideri Rehd.). Genome Res. 2013;23(2):396-408.

18. Crocco CD, Botto JF. BBX proteins in green plants: insights into their evolution, structure, feature and functional diversification. Gene. 2013:531(1):44-52.

19. Cockram J, Thiel T, Steuernagel B, Stein N, Taudien S, Bailey PC, O'Sullivan DM. Genome dynamics explain the evolution of flowering time CCT domain gene families in the Poaceae. PLoS One. 2012;7(9):e45307.

20. Gao Y, Zhou H, Chen J, Jiang X, Tao S, Wu J, Zhang S. Mitochondrial dysfunction mediated by cytoplasmic acidification results in pollen tube growth cessation in Pyrus pyrifolia. Physiol Plant. 2015;153(4):603-15.

21. Chu Z, Wang X, Li Y, Yu H, Li J, Lu Y, Li H, Ouyang B. Genomic organization, phylogenetic and expression analysis of the B-BOX gene family in tomato. Front Plant Sci. 2016:7(10):1552.

22. Huang J, Zhao X, Weng X, Wang L, Xie W. The rice B-box zinc finger gene family: genomic identification, characterization, expression profiling and diurnal analysis. PLoS One. 2012;7(10):e48242. 
23. Yu J, Hu S, Wang J, Wong GK-S, Li S, Liu B, Deng Y, Dai L, Zhou Y, Zhang X. A draft sequence of the rice genome (Oryza sativa L. ssp. indica). Science. 2002;296(5565):79-92.

24. Initiative AG. Analysis of the genome sequence of the flowering plant Arabidopsis thaliana. Nature. 2000;408(6814):796.

25. Consortium TG. The tomato genome sequence provides insights into fleshy fruit evolution. Nature. 2012;485(7400):635-41.

26. Moore RC, Purugganan MD. The early stages of duplicate gene evolution. Proc Natl Acad Sci. 2003;100(26):15682-7.

27. Cao Y, Han Y, Meng D, Li D, Jin Q, Lin Y, Cai Y. Structural, evolutionary, and functional analysis of the class III peroxidase gene family in Chinese Pear (Pyrus bretschneideri). Front Plant Sci. 2016;7(10):1874.

28. Cao Y, Han Y, Li D, Lin Y, Cai Y. Systematic analysis of the 4-Coumarate: Coenzyme A Ligase $(4 \mathrm{CL})$ related genes and expression profiling during fruit development in the Chinese Pear. Genes. 2016;7(10):89.

29. Cao Y, Han Y, Meng D, Li G, Li D, Abdullah M, Jin Q, Lin Y, Cai Y. Genomewide analysis suggests the relaxed purifying selection affect the evolution of WOX genes in Pyrus bretschneideri, Prunus persica, Prunus mume, and Fragaria vesca. Front Genet. 2017;8(2):78.

30. Han Y, Ding T, Su B, Jiang H. Genome-wide identification, characterization and expression analysis of the Chalcone Synthase Family in Maize. Int J Mol Sci. 2016;17(2):161.

31. Cannon SB, Mitra A, Baumgarten A, Young ND, May G. The roles of segmental and tandem gene duplication in the evolution of large gene families in Arabidopsis thaliana. BMC Plant Biol. 2004;4(1):10.

32. Gangappa SN, Holm M, Botto JF. Molecular interactions of BBX24 and BBX25 with HYH, HY5 HOMOLOG, to modulate Arabidopsis seedling development. Plant Signaling \& Behavior. 2013;8(8):e25208.

33. Cai Y, Li G, Nie J, Lin Y, Nie F, Zhang J, Xu Y. Study of the structure and biosynthetic pathway of lignin in stone cells of pear. Sci Hortic. 2010;125(3): 374-9.

34. Jin Q, Yan C, Qiu J, Zhang N, Lin Y, Cai Y. Structural characterization and deposition of stone cell lignin in Dangshan Su pear. Sci Hortic. 2013;155: $123-30$

35. Zdobnov EM, Apweiler R. InterProScan-an integration platform for the signature-recognition methods in InterPro. Bioinformatics. 2001;17(9):847-8.

36. Punta M, Coggill PC, Eberhardt RY, Mistry J, Tate J, Boursnell C, Pang N, Forslund K, Ceric G, Clements J. The Pfam protein families database. Nucleic Acids Res. 2011:40(D1):D290-301.

37. Letunic I, Doerks T, Bork P. SMART 7: recent updates to the protein domain annotation resource. Nucleic Acids Res. 2012;40(D1):D302-5.

38. Tamura K, Peterson D, Peterson N, Stecher G, Nei M, Kumar S. MEGA5: molecular evolutionary genetics analysis using maximum likelihood, evolutionary distance, and maximum parsimony methods. Mol Biol Evol. 2011;28(10):2731-9.

39. Crooks GE, Hon G, Chandonia J-M, Brenner SE. WebLogo: a sequence logo generator. Genome Res. 2004;14(6):1188-90.

40. Hu B, Jin J, Guo YA, Zhang H, Luo J, Gao G. GSDS 2.0: an upgraded gene feature visualization server. Bioinformatics. 2014;31(8):1296.

41. Wang Y, Tang H, DeBarry JD, Tan X, Li J, Wang X, Lee T-H, Jin H, Marler B, Guo H. MCScanX: a toolkit for detection and evolutionary analysis of gene synteny and collinearity. Nucleic Acids Res. 2012;40(7):e49.

42. Wang D, Zhang Y, Zhang Z, Zhu J, Yu J. Kaks_Calculator 2.0: a toolkit incorporating gamma-series methods and sliding window strategies. Genomics Proteomics Bioinformatics. 2010:8(1):77-80.

43. Zhou H, Qi K, Xing L, Hao Y, Peng W, Chen J, Wu J, Zhang S. Genome-wide identification and comparative analysis of the cation proton antiporters family in pear and four other Rosaceae species. Mol Gen Genomics. 2016; 291(4):1727.

44. Zhou H, Yin H, Chen J, Liu X, Gao Y, Wu J, Zhang S. Gene-expression profile of developing pollen tube of Pyrus bretschneideri. Gene Expr Patterns. 2016; 20(1):11-21.

45. Muthamilarasan M, Khandelwal R, Yadav CB, Bonthala VS, Khan Y, Prasad M. Identification and molecular characterization of MYB transcription factor superfamily in C 4 model plant foxtail millet (Setaria italica L.). PLoS One. 2014;9(10):e109920.

46. Wu T, Zhang R, Gu C, Wu J, Wan H, Zhang S, Zhang S. Evaluation of candidate reference genes for real time quantitative PCR normalization in pear fruit. Afr J Agric Res. 2012;7:3701-4.

47. Livak KJ, Schmittgen TD. Analysis of relative gene expression data using realtime quantitative PCR and the 2- $\Delta \Delta C T$ method. Methods. 2001;25(4):402-8.
48. Chung E, Seong E, Kim YC, Chung EJ, Oh SK, Lee S, Park JM, Joung YH, Choi D. A method of high frequency virus induced gene silencing in chili pepper (Capsicut annuит L. cv. Bukang). Mol Cells. 2004;17(2):377-80.

49. Sparkes IA, Runions J, Kearns A, Hawes C. Rapid, transient expression of fluorescent fusion proteins in tobacco plants and generation of stably transformed plants. Nat Protoc. 2006;1(4):2019-25.

\section{Submit your next manuscript to BioMed Central and we will help you at every step:}

- We accept pre-submission inquiries

- Our selector tool helps you to find the most relevant journal

- We provide round the clock customer support

- Convenient online submission

- Thorough peer review

- Inclusion in PubMed and all major indexing services

- Maximum visibility for your research

Submit your manuscript at www.biomedcentral.com/submit
) Biomed Central 